\title{
Misjudging where you felt a light switch in a dark room
}

\author{
Femke Maij • Denise D. J. de Grave • \\ Eli Brenner · Jeroen B. J. Smeets
}

Received: 14 September 2010/Accepted: 5 April 2011/Published online: 23 April 2011

(c) The Author(s) 2011. This article is published with open access at Springerlink.com

\begin{abstract}
Previous research has shown that subjects systematically misperceive the location of visual and haptic stimuli presented briefly around the time of a movement of the sensory organ (eye or hand movements) due to errors in the combination of visual or tactile information with proprioception. These briefly presented stimuli (a flash or a tap on the finger) are quite different from what one encounters in daily life. In this study, we tested whether subjects also mislocalize real (static) objects that are felt briefly while moving ones hand across them, like when searching for a light switch in the dark. We found that subjects systematically mislocalized a real bar in a similar manner as has been shown with artificial haptic stimuli. This demonstrates that movement-related mislocalization is a real world property of human perception.
\end{abstract}

Keywords Haptics · Mislocalization · Human

\section{Introduction}

When you enter a room late at night and need to find the light switch in total darkness, you sweep your hand across the wall to feel where the switch is. During these sweeping movements, you need to know where your hand is in space (information provided by proprioception and motor commands), and you need to know whether and when your hand feels the switch (tactile information). The tactile information has to be combined with information about

F. Maij ( $₫)$ · D. D. J. de Grave · E. Brenner · J. B. J. Smeets Research Institute MOVE, Faculty of Human Movement Sciences, VU University, Van der Boechorststraat 9, 1081, BT, Amsterdam, The Netherlands

e-mail: femke.maij@gmail.com; f.maij@bham.ac.uk posture in order for you to know where the light switch is located. Timing is critical when combining the two senses (touch and proprioception), because the light switch is located where the hand was at the time it touched the switch. Once you have moved over the switch, you need to move your hand back to the place at which you felt it to turn on the light. Surprisingly, it is hard to find the switch although you just touched it while moving your arm. In this paper, we study this localization problem, especially whether these localization errors are systematic.

In vision, it is known that people make systematic errors when localizing objects flashed around the time of saccades (Matin and Pearce 1965; Bischof and Kramer 1968; Mateeff 1978; Dassonville et al. 1992; Ross et al. 1997; Lappe et al. 2000; Lavergne et al. 2010; Maij et al. 2011a) or during smooth pursuit (Brenner et al. 2001; Rotman et al. 2004; Kerzel et al. 2006). These systematic localization errors are primarily in the direction parallel to the movement (Honda 1993). Similar mislocalization patterns have been reported in haptics (Dassonville 1995; Watanabe et al. 2009). In those studies, a small vibrator, attached to the index finger, delivered a tap on the finger around the time of an arm movement.

All the above-mentioned studies used artificial stimuli that differ in many ways from what we normally encounter in our everyday environment. In vision, very short flashes were presented, and in haptics, the finger was tapped with no displacement on the skin. Dassonville (1995) raised the question in the discussion of his paper as to why these large haptic mislocalizations are not more obvious in daily life. In the present study, we tested whether the same pattern of mislocalization occurs under more natural conditions. Subjects were instructed to localize a thin bar that was placed on top of a table. They felt this bar while moving their arm from one location to another across the table. We 
will show that this task, which is comparable to the lightswitch example mentioned above, yields similar systematic errors to those found with artificial stimuli.

\section{Methods}

Six subjects volunteered to participate in the experiment (including two of the authors). The study is part of a research program that was approved by the ethics committee of the Faculty of Human Movement Sciences.

\section{Setup}

We instructed the subjects to sit on a chair and blindfolded them. We asked the subjects to move their right index finger either to the left or to the right across a tabletop (the two most distal phalanges of the index finger were placed on the tabletop) from the side of one cube (sides: $2.5 \mathrm{~cm}$ ) to the side of another cube (see Fig. 1a). The space between the cubes was $40 \mathrm{~cm}$. We placed a thin aluminum bar ( $5 \mathrm{~mm}$ wide; $1 \mathrm{~mm}$ high) on the table at a random position on the movement path. The bar was oriented orthogonally to the path and was long enough for its far end never to be felt. The right index finger's trajectory was recorded by attaching an infrared light-emitting diode (IRED) to the nail and recording the IRED's position with an Optotrak system (Northern Digital Incorporation, Waterloo, Ontario, Canada; sampling rate $=500 \mathrm{~Hz}$ ). Three additional IRED's were attached to the aluminum bar and the two cubes.

\section{Procedure}

Each trial started by the experimenter instructing the subject at which cube to start. This was determined at random.
The subject placed the index finger so that it touched either the left side of the cube on the right or the right side of the cube on the left. The bar was then placed at a random position between the cubes and the subject was instructed to start. He or she moved his or her fingertip across the surface of the table at whatever speed he or she found convenient until the finger reached the other cube. After this movement, the subject had to indicate the perceived location of the bar by lifting the finger and placing it at that location, see Fig. 1b. When the subject started moving to the perceived location of the bar, the researcher moved the bar to another location along the path so that the subjects received no feedback (subjects were aware of this). In total, there were 160 trials for each subject (80 trials starting at each side).

\section{Data analysis}

Following Dassonville (1995), and because our findings in vision suggest that the mislocalization has a temporal origin (Maij et al. 2009, 2011b), we express the errors as timing errors. We used the recorded positions of the index finger and bar to determine the localization error and the corresponding timing error (as explained below and in Fig. 1b). The beginning and end of the movement were determined with a velocity threshold of $5 \mathrm{~cm} / \mathrm{s}$. We discarded trials in which the arm movement was not smooth (for instance because the subject stopped moving the finger after he or she crossed the bar) and trials in which the subject started moving before the instruction to start.

Positive values of the localization error indicate an error in the direction of the arm movement (nearer to the end position of the movement, e.g., indicating a position that is too far to the right for a rightward movement). We plotted the localization error as a function of the different locations and times of contact with the bar (relative to movement

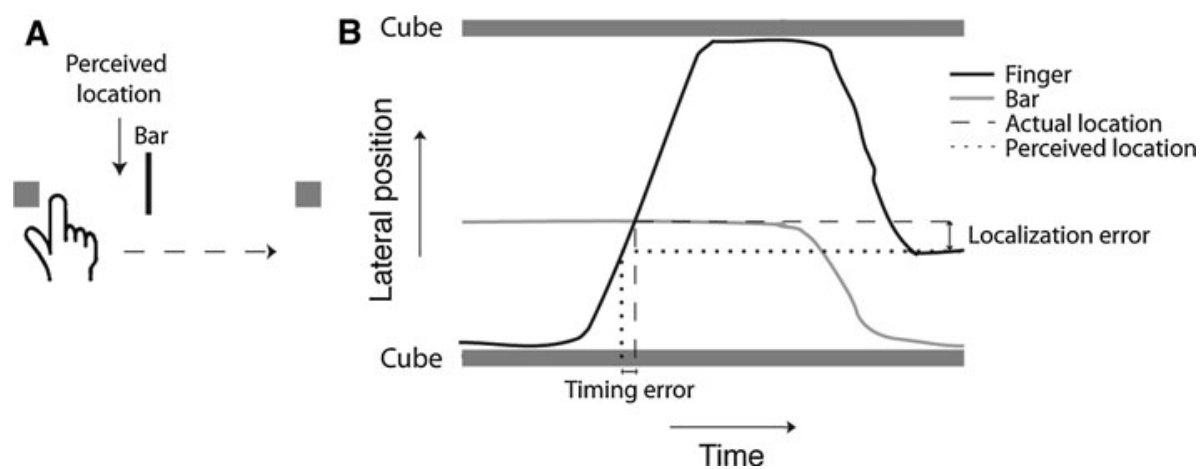

Fig. 1 The haptic localization task. a Top view. b Time course of an example trial. The subject moved the fingertip (black line) from the left cube to the right one. During the movement, the finger crossed the aluminum bar (gray line). After reaching the other cube, the finger moved back to the perceived location of the bar (that had been moved away in the meantime). The movement to the perceived location of the bar was performed with the finger lifted off the table. The difference between the perceived location and the actual (original) location of the bar is the localization error. The timing error is the difference in time between when the finger reached the actual location of the bar and when it reached the perceived location of the bar (during the initial movement) 
onset). The timing error was defined as the difference in time between when the finger reached the actual location and when it reached the perceived location of the bar (see Fig. 1b). We fit a regression line through the data points of the timing error to compare the results with those of Dassonville (1995).

The duration of the contact between the finger and the bar was determined by dividing the sum of the width of the finger (approximately $1.5 \mathrm{~cm}$ ) and the width of the static bar $(0.5 \mathrm{~cm})$ by the velocity of the finger's movement at the time of contact. The average duration of contact was determined for each subject, as was the average peak velocity of the finger.

\section{Results}

On average, we discarded $3 \%$ of the trials. The average duration of contact between the finger and the bar was $28 \pm 7 \mathrm{~ms}$ (mean \pm standard deviation across subjects). For each subject, we display the perceived distance of the bar from the start position as a function of the actual distance (Fig. 2a). Three of the six subjects (EB, AH, and WS) have a tendency to underestimate the distance. All subjects have considerable variability in where they localize the bar when it is presented at any given position.
This variability (standard deviations of several $\mathrm{cm}$ ) is considerably larger than the reported precision of (static) proprioceptive localization at similar positions (van Beers et al. 1998).

Subjects differed significantly in the average velocity at which they moved their finger $(P<0.001)$. This was mainly due to subject ND who moved almost twice as fast as the average of the other subjects (mean and standard deviation of the velocity of ND was $124 \pm 16 \mathrm{~cm} / \mathrm{s}$, whereas that of the other subjects ranged from $52 \pm 9$ to $83 \pm 14 \mathrm{~cm} / \mathrm{s}$ ). By dividing the data into three epochs and comparing the start of the session with the end of the session, we found that the accuracy, variability, and mean velocity of individual subjects did not differ significantly ( $P=0.8, P=0.3$ and $P=0.5$, respectively).

In Fig. 2b, we show the localization errors as a function of the time from movement onset. The negative slope of the smooth curve through the data points indicates that the longer the finger moved, the more the bar was perceived at a position that was crossed earlier than was the actual bar location. This is even more evident from the timing error (the time between when the finger was at the actual location and when the finger was at the perceived location; Fig. 2c). One subject shows a different result (ND). This subject moved very fast and the bar was usually perceived to be near either the start position or the end position of the
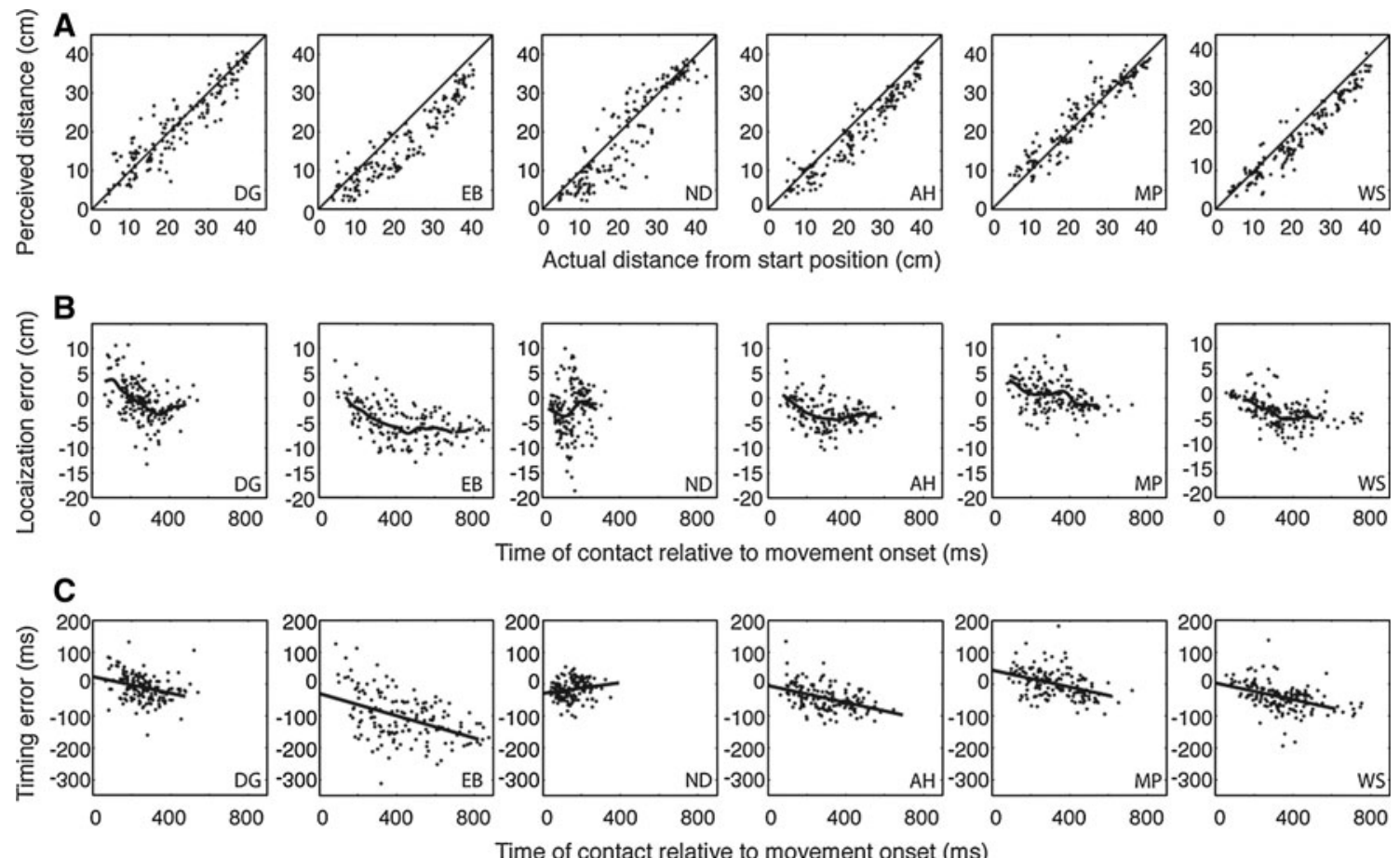

Fig. 2 Each subject's performance. a The perceived position of the bar, as a function of the actual position of the bar. Solid line represents veridical localization of the bar. $\mathbf{b}$ The localization error, as a function of how long after movement onset the finger touched the bar. The smooth curves through the dots are averages based on a moving Gaussian window (filled circle $=25 \mathrm{~ms}$ ). $\mathbf{c}$ Timing error as a function of how long after movement onset the finger touched the bar. The lines represent linear regressions to the data points 
Table 1 Regression coefficients of linear fits of the timing error as a function of the time of contact with the bar relative to movement onset. We also give the ranges of the intercept, slope, and $R^{2}$ values of the four subjects from table 3 of Dassonville (1995)

\begin{tabular}{llll}
\hline Subject & \multicolumn{3}{l}{ Regression coefficient of the timing error } \\
\cline { 2 - 4 } & Intercept $(\mathrm{ms})$ & Slope & $R^{2}$ \\
\hline DG & 23 & $-0.13^{* *}$ & 0.10 \\
EB & -29 & $-0.17^{* *}$ & 0.22 \\
ND & -29 & $0.09^{*}$ & 0.04 \\
AH & -5 & $-0.13^{* *}$ & 0.19 \\
MP & 44 & $-0.13^{* *}$ & 0.17 \\
WS & 4 & $-0.13^{* *}$ & 0.19 \\
Mean & 1 & -0.10 & \\
Dassonville & 72 to 162 & -0.12 to -0.49 & 0.11 to 0.73 \\
\hline
\end{tabular}

* Slope is significantly greater than zero $(t$ test, $P<0.05)$

** Slope is significantly less than zero $(t$ test, $P<0.0001)$

movement. Regression coefficients for the timing error as a function of the time of contact relative to movement onset are shown in Table 1.

\section{Discussion and conclusion}

We show that subjects make similar systematic errors when localizing an object that they touch while making an arm movement in conditions that could occur in daily life and as have previously been found when tested under more artificial conditions (a tap on a moving finger; (Dassonville 1995; Watanabe et al. 2009). This finding is particularly important because the comparable systematic localization errors in vision have also only been demonstrated for quite artificial stimuli: extremely short flashes.

We found a smaller average slope of the regression lines of the timing error as a function of time relative to movement onset than the slope of the regression lines in the experiment of Dassonville (1995). This difference in slope could arise from the fact that the average duration of the tactile stimulus was clearly longer in our experiment (28 ms) than the $6 \mathrm{~ms}$ in the study of Dassonville (1995), based on analogous results in vision. Visual experiments that show considerable systematic mislocalization have in general been performed with extremely short flashes (e.g., Schlag and Schlag-Rey 1995; Ross et al. 1997; Lappe et al. 2000). Rotman et al. (2005) showed that such systematic localization errors decrease with increasing stimulus duration for flashes presented during smooth eye movements.

The intercepts of the regression lines of the timing error are smaller than the intercepts found by Dassonville
(1995). This could be explained by one or more of the other differences between the studies. In Dassonville's haptic localization study, the stimulus could be presented before, during, and after the start of the hand movement. In our experiment, subjects had to move their finger to touch the bar, so we could only use locations of the bar that the finger crossed during the movement. This resulted in a smaller range of positions at which the bar could be presented. Moreover, Dassonville's subjects moved their hand in the air (obtaining only proprioceptive information about the location of the finger), whereas in our study the subjects were instructed to move the finger across a surface (obtaining both proprioceptive and cutaneous information about the location of the finger).

In summary, we can conclude that errors when touching a real object are not fundamentally different from ones when the stimulus is delivered by a vibrator attached to the finger. This demonstrates that movement-related mislocalization is not limited to artificial stimuli. We show that these errors are present in a task that is reminiscent of finding a light switch in the dark.

Acknowledgments We thank Frans-Josef Halkes for technical support. This research was supported by the Netherlands Organization for Scientific Research (NWO, ALW grant 816-02-017).

Open Access This article is distributed under the terms of the Creative Commons Attribution Noncommercial License which permits any noncommercial use, distribution, and reproduction in any medium, provided the original author(s) and source are credited.

\section{References}

Bischof N, Kramer E (1968) Investigations and considerations of directional perception during voluntary saccadic eye movements. Psychol Forsch 32:185-218

Brenner E, Smeets JBJ, van den Berg AV (2001) Smooth eye movements and spatial localisation. Vis Res 41:2253-2259

Dassonville P (1995) Haptic localization and the internal representation of the hand in space. Exp Brain Res 106:434-448

Dassonville P, Schlag J, Schlag-Rey M (1992) Oculomotor localization relies on a damped representation of saccadic eye displacement in human and nonhuman primates. Vis Neurosci 9:261-269

Honda H (1993) Saccade-contingent displacement of the apparent position of visual stimuli flashed on a dimly illuminated structured background. Vis Res 33:709-716

Kerzel D, Aivar MP, Ziegler NE, Brenner E (2006) Mislocalization of flashes during smooth pursuit hardly depends on the lighting conditions. Vis Res 46:1145-1154

Lappe M, Awater H, Krekelberg B (2000) Postsaccadic visual references generate presaccadic compression of space. Nature 403:892-895

Lavergne L, Vergilino-Perez D, Lappe M, Dore-Mazars K (2010) The spatial pattern of peri-saccadic compression for small saccades. J Vis 10(14):17.11-17.12

Maij F, Brenner E, Smeets JBJ (2009) Temporal information can influence spatial localization. J Neurophysiol 102:490-495 
Maij F, Brenner E, Smeets JBJ (2011a) Peri-saccadic mislocalization is not influenced by the predictability of the saccade target location. Vis Res 51:154-159

Maij F, Brenner E, Smeets JBJ (2011b) Temporal uncertainty separates flashes from their background during saccades. J Neurosci 31(10):3709-3711

Mateeff S (1978) Saccadic eye movements and localization of visual stimuli. Percept Psychophys 24:215-224

Matin L, Pearce DG (1965) Visual perception of direction for stimuli flashed during voluntary saccadic eye movements. Science 148:1485-1488

Ross J, Morrone MC, Burr DC (1997) Compression of visual space before saccades. Nature 386:598-601
Rotman G, Brenner E, Smeets JBJ (2004) Mislocalization of targets flashed during smooth pursuit depends on the change in gaze direction after the flash. J Vis 4:4.564-4.574

Rotman G, Brenner E, Smeets JBJ (2005) Flashes are localised as if they were moving with the eyes. Vis Res 45:355-364

Schlag J, Schlag-Rey M (1995) Illusory localization of stimuli flashed in the dark before saccades. Vis Res 35:2347-2357

van Beers RJ, Sittig AC, Denier van der Gon JJ (1998) The precision of proprioceptive position sense. Exp Brain Res 122:367-377

Watanabe J, Nakatani M, Ando H, Tachi S (2009) Haptic localizations for onset and offset of vibro-tactile stimuli are dissociated. Exp Brain Res 193:483-489 